\title{
Methionine Metabolism in Humans: New Perspectives on Epigenetic Inheritance
}

\section{Venkatachalam KV}

College of Medical Sciences, Nova Southeastern University, USA

*Corresponding author: Venkatachalam KV, Professor of Biochemistry, College of Medical Sciences, Nova Southeastern University, Ft. Lauderdale, FL-33328, USA, Tel: (954)262-1335; E-mail: venk@nova.edu

Rec date: August 13, 2014; Acc date: October 14, 2014; Pub date: October 16, 2014

Copyright: (c) 2014 Venkatachalam KV. This is an open-access article distributed under the terms of the Creative Commons Attribution License, which permits unrestricted use, distribution, and reproduction in any medium, provided the original author and source are credited.

\begin{abstract}
Epigenetic control by methylation is directly dependent on SAM/methionine. Free methionine aside from being used for protein synthesis is activated to universal methyl group donor SAM and the methyl group from it can be transferred mainly onto DNA, histones, mRNA and noncoding (Nc) RNA, in the nucleus and onto various cytosolic recipients by specific methyltransferases. The product S-adenosylhomocysteine (SAH) from methyltransferase reaction is cleaved into free homocysteine and adenosine. Homocysteine has two fates, one to enter methionine resynthesis which requires methionine synthase (MetS), the coenzymes N5-methyltetrahydrofolate (vitamin B9) and methylcobalamin (vitamin B12). The second option of homocysteine is to enter cysteine synthesis pathway which requires cystathionine -synthase (CBS), serine, vitamin B6 (pyridoxal phosphate) and cystathionine $\mathrm{Y}$-lyase (CGL). I predict serine and vitamin B6 are the critical diverting factors in a fully developed organism where the methylation is minimal and all other factors such as MetS, B9, B12, ATP, Methionine adenosyltransferase (MAT), are more relevant during normal development or in abnormal deregulated cancer cell metabolism. In a developed organism the demethylation pathway would play a critical role during tissue regeneration and must involve hydroxylation $(\mathrm{CH} 2 \mathrm{OH})$, oxidation $(\mathrm{CHO})$ and decarboxylation $(\mathrm{COOH})$ and the corresponding hydroxylase, oxidase and decarboxylase. The hydroxylation is predicted to involve vitamin $\mathrm{C}$ (ascorbate) and decarboxylation would involve (vitamin B6). The control of methylation and equally the demethylation is extremely crucial for epigenetic control mechanisms (gene suppression/expression) and any dysregulation would cause abnormal tissues/cancer.
\end{abstract}

Keywords: Methionine; S-adenosylmethionine (SAM); 5methylcytosine; Biochemistry of methylation/demethylation; Epigenetic control by methylation/demethylation

\section{Perspectives}

The element sulfur with an atomic number of 16 and a mass of $\sim 32.07$ had been incorporated in various organisms during the course of evolution and it occurs in different redox forms. In mammals, one form of sulfur is obtained as sulfate anion which is transported via several tissue specific carrier proteins that are located on the plasma membranes [1]. Once inside the cell, sulfate anion is converted into universal sulfonate (sulfuryl) donor 3'phosphosdenosine 5'phosphosulfate (PAPS) in two steps catalyzed by ATP sulfurylase and APS kinase activities of PAPS synthase (PAPSS) [2]. There are different isozymes and isoforms (splice variants) of PAPSS in humans [3]. The transfer of sulfonate group from PAPS onto a recipient sugar, protein or lipid is catalyzed by several specific sulfotransferases (SULT's) [4]. The sulfate of the recipient compound would receive1 negative charge. In contrast, the kinases transfer phosphoryl group from ATP on to recipient molecule to form a phosphate group which would bear 2 negative charges. Beside the charge differences between phosphate and sulfate group on the recipient molecule and very minor bond length (tetrahedral phosphate, $\mathrm{P}=\mathrm{O} \sim 152 \mathrm{pm}$ ) (tetrahedral sulfate $\mathrm{S}=\mathrm{O} \sim 149 \mathrm{pm}$ ), what evolutionary advantage it may impart is a paradigm except we can conceive that the system had added additional variety to the metabolic pool. The reduction of sulfate to sulfide doesn't happen in humans (mammals), instead humans obtain the most reduced sulfide in the form of sulfur amino acid cysteine [5]. Cysteine is required for tripeptide ( $\gamma$-glutamyl- Cys-Gly), glutathione (GSH) synthesis. GSH is the cells reducing agent that controls intracellular red/ox states [6]. It is the thiol, a reduced functional group of cysteine that participates in cells redox reactions functioning in the form of glutathione [6] or thioredoxin protein [7]. Cysteine is also used in many proteins during synthesis. During the course of these redox reactions cysteine gets oxidized to cystines (the peptide or protein disulfide). Free cysteine can also be oxidized in tissues like brain to form taurine (2-aminoethylsulfonate) which serves as a neurotransmitter [8].

Metabolic draining of cysteine into GSH metabolism due to high demands of the xenobiotic detoxification by glutathione s-transferase can lead to reduced methionine pools. This in turn can reduce overall methylation of epigenomes and increase the risk for cancer [9]. Besides cysteine, the other form of sulfur that humans can intake through diet is methionine. Methionine is a sulfur amino acid which has carbon-sulfur-methyl thioether bond [10]. Methionine is activated to s-adenosylmethionine (SAM) using ATP as an adenosyl donor by methionine adenosyltransferase (EC 2.5.1.6). SAM is the universal methyl group donor which bears a positive charge on the sulfur $[11,12]$. The role of SAM in histone, DNA and mRNA methylation is extremely crucial in epigenetic regulations of genome/gene expressions [13]. Once the methyl group is transferred to a recipient molecules such as nucleic acids, lipids, proteins and sugars the side product s-adenosylhomocysteine ( $\mathrm{SAH}$ ) is cleaved into homocysteine and adenosine. SAH is a potent inhibitor of methyltransferases and $\mathrm{SAH}$ is cleaved into adenosine and homocysteine by SAH hydrolase [14]. Free homocysteine can take two pathways either to enter cysteine synthesis or the methionine resynthesis. For cysteine synthesis: homocysteine first condenses with serine to form cystathionine with the elimination of $\mathrm{H}_{2} \mathrm{O}$, catalyzed by pyridoxal phosphate [PLP, (coenzyme derivative of vitamin B6)] dependent cystathionine 
synthase (CBS) (E.C. 4.21.22) [15]. Cystathionine -lyase (CGL) (E.C. 4.4.1.1) [16], a trans-sulfurylase using PLP cleaves cystathionine at the $\gamma$-position to release cysteine and the rest of the moiety is deaminated to form a-ketobutyrate and ammonia. For methionine resynthesis: the free homocysteine is converted into methionine by methionine synthase [5-methyltetrahydropteroyl-L-glutamate: L-homocysteine smethyltransferase, (E.C. 2.1.1.13)] using methyl group from 5-methyltetrahydrofolate and the coenzyme methylcobalamin (vitamin B12) [17]. Methionine synthase is the only enzyme that uses both vitamin B9 (in the form of methyl-tetrahydrofolate) and vitamin B12 (in the form of methylcobalamin) [18]. The other only enzyme that uses vitamin $\mathrm{B} 12$ in the form of adenosylcobalamin is methylmalonyl CoA mutase. Thus, the importance of coenzyme forms of both vitamin B9 (folate) and B12 (cobalamin) is extremely crucial for endogenous synthesis of methionine. Whereas it seems like vitamin B6 and especially serine are important factors in diverting homocysteine to cysteine synthesis. From free methionine s-adenosylmethionine (SAM) is synthesized using ATP by methionine adenosyltransferase (MAT). There are cytosolic and nuclear MAT's which can partition the SAM between cytosol and nucleus. The MAT II is the nuclear isoform which can serve as a transcriptional corepressor for Maf oncoprotein [19]. In the cytosol the tri-methylation of phosphatidylethanolamine to form phosphatidylcholine and endogenous formation of carnitine from trimethyl-lysine are the main methylation reactions that happen in most tissues. In some specialized tissues of brain, epinephrine synthesis from norepinephrine and degradation of catecholamine involves $\mathrm{N}$ and $\mathrm{O}$ methylation. In contrast to cytosol, the methylation metabolism in the nucleus mainly happens to methylate DNA, histones and NcRNA, mRNA 5'Gcapping, which are all very active during development. In contrast the reverse process of demethylation would dominate during tissue regeneration and during developmental patterning and reprogramming. Abnormal methylation patterns can cause metabolic deregulation and eventual cancer. The disruption of cytosolic and/or nuclear methionine pools and its effects on specific methylation defects are under investigation by transfection of bacterial methionine degrading enzyme (MGLD) an enzyme that is absent in mammals [20], Venkatachalam et. al. unpublished]. This would help unravel methionine deprivation induced changes in cancer/normal cell metabolism and growth. Under abnormal conditions due to low levels of endogenous coenzymes B9, B12 and/or due to low activities of methionine synthase (mutant form), free homocysteine can nonspecifically form homocysteinethiolactone (a cyclic ester) through low fidelities of cysteinyl tRNA synthetase. Homocysteine thiolactone is very reactive compound that can form modified LDL which eventually can aggregate and lead to atherosclerotic plaques. In addition homocysteine can be channeled for cysteine synthesis when the levels of B6, serine, CBS and CGL are appropriate. If there is a severe defect in any one of the two pathways of homocysteine metabolism it is sufficient to cause homocysteinemia [21]. Thus, the dynamic regulation of sulfur amino acid pool is extremely crucial for normal cell metabolism. The disruption of this metabolism could lead to various problems such as redox imbalance or methylation defect. In brain this can lead to autism spectrum of diseases, psychiatric disorders, neurodegenerative and other related diseases. The molecular developmental gene expressions of various enzymes described above would control the overall methylations patterns of histones, DNA and mRNA which then would control the expressions/ suppressions of genes that are under morphogenetic and environmental cues/regimens [22-24]. The dietary practices and intakes of the key sulfur amino acids can also influence intracellular pools of these metabolites which in turn can control the epigenetic patterns and epigenetic circuits of neuron potentiation and reinforcements. This dietary influence can affect various cell types on its metabolism and epigenetics [25]. The three key behavioral elements of life: acceptance, avoidance and contradictions are reinforced by short term and long term mechanisms and they are perhaps controlled at the epigenetic levels by methylation patterns/circuits. The nucleotide cytosine is often methylated at the 5 th carbon by specific methyltransferase. Under certain conditions 5-methylcytosine can undergo deamination at the 4 th position and can lead to the formation of deoxythymine (dT). This can then result in aberrant molecular pattern formation at the epi level of DNA due to newly formed dT residues.

However, under normal conditions at the epigenetic level the $5 \mathrm{mC}$ ("zeroes") and the de/unmethylated cytosine ("ones") can perhaps serve as epigenetic codes. Thus in an analogy one could envision what computer codes are made of: the zeroes and ones. Whether the formation of $\mathrm{dT}$ happens naturally or due to mutagen induced deaminase expression is not very clear. Nevertheless the expression and the regulation of deaminase due to environmental stimuli or during developmental patterning are very significant. The removal of dT base pair happens through the activities of base pair excision $[26,27]$. Though equally important the process of demethylation is poorly understood. Biochemically demthylation of intact methyl group is difficult. However, upon hydroxylation, and further oxidation into formyl and carboxyl it can be removed easily. The original methyl group now in the form of labile carboxyl group can be decarboxylated by coenzyme PLP, dependent decarboxylase(s) (Venkatachalam unpublished). The hydroxylation of 5-methylcytosine to form 5-hmC catalyzed by specific hydroxylases is a reaction that is part of the methyl erasing process which I predict would require vitamin C ascorbate. The direct role of activated methionine, SAM in methylation (methyl writing) is extremely crucial in epigenetic control mechanisms. The methyl group recognition by various transcription factors (methyl reading) is very crucial for gene expression patterns that are cell/tissue specific. Demethylation (methyl erasing) of histones, DNA and NcRNA after hydroxylation, oxidation and decarboxylation could be a normal process during fetal development, growth and adult tissue regeneration. However, inappropriate methylation (writing), methyl recognition (reading) and demethylation (erasing) due to environmental pollutants and toxins can lead to alterations and eventual disease manifestations such as cancers, neurological degenerations and various debilitating diseases. Thus, the fine balance of keeping the methylated (suppressed)/ demethylated (expressed) states of histones, DNA and NcRNA are fundamental in maintaining normal biology of cell. Deregulation/ dysregulation in this fine balance could alter the nature of the organismal physiology [28].

\section{Conclusions}

Methylation/demethylation pathways are critical in controlling the epigenetic mode of gene suppression/expression. For methylation: MetS, CBS, SAM synthase, SAH, vitamin B9, vitamin B12, methyltransferases, methionine nuclear transporter, ATP and MAT are required to tightly regulate the cycle (Figure 1). Although wealthy of information is available regarding methylation the process of demethylation is poorly understood. For demethylation: cytosine methyl hydroxylase (purported vitamin $\mathrm{C}$ dependent enzyme), oxidase and the decarboxylase must be tightly regulated. The vitamin B6 
dependent cytosine 5-decarboxylase is newly proposed and poorly understood enzyme that needs to be characterized thoroughly (Venkatachalam unpublished). Cytosine 5-decarboxylase would be controlled developmentally, temporally and chronologically. De/ dysregulated activity of cytosine 5-decarboxylase would result in uncontrolled cell division and metabolism (Figure 2).

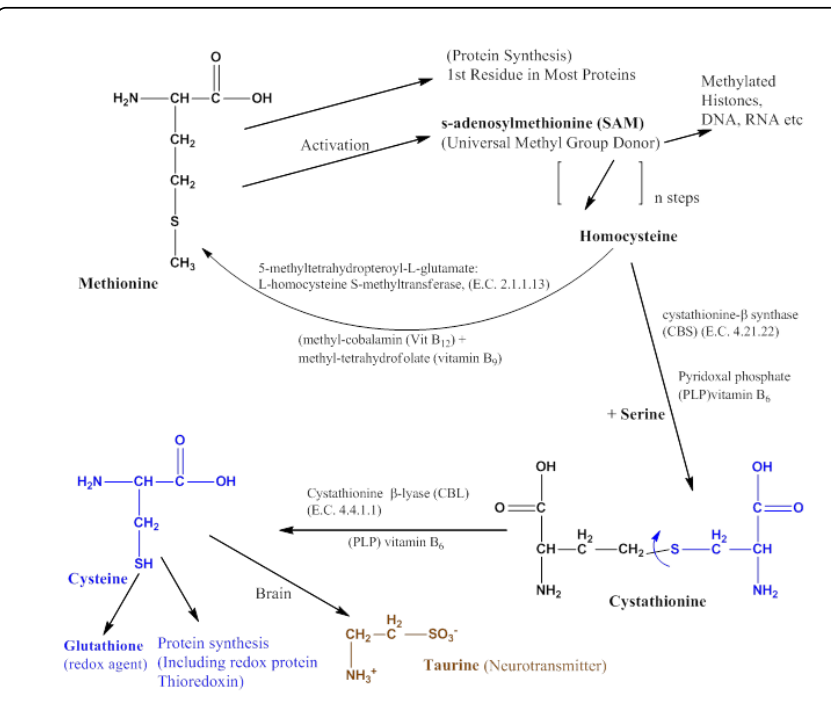

Figure 1: Methionine metabolism. Serine/CBS control over resynthesis of methionine or de novo cysteine synthesis. Neurotransmitter taurine synthesis is specialized in brain

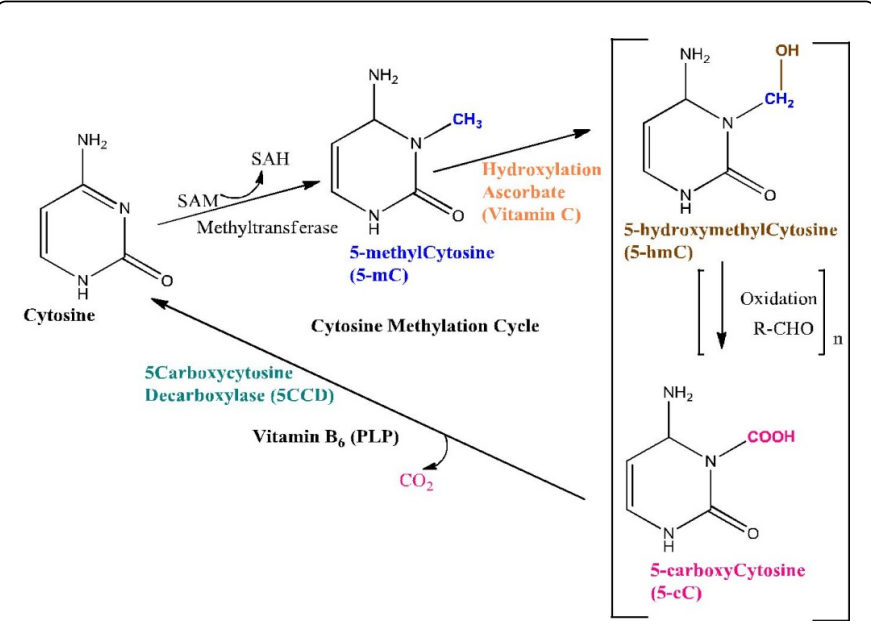

Figure 2: Cytosine methylation and demethylation pathway. Purported role of 5-carboxycytosine decarboxylase is emphasized ( Venkatachalam). Molecular targets and epigenetic medicine can be designed based on the pathway that is described.

\section{References}

1. Markovich D (2001) Physiological roles and regulation of sulfate transporters. Phys Rev 81: 1499-1533.

2. Venkatachalam KV, Akita H, Strott CA (1998) Molecular cloning, expression, and characterization of human bifunctional 3'- phosphoadenosine 5'-phosphosulfate synthase and its functional domains. J Biol Chem 273: 19311-19320.

3. Venkatachalam KV (2003) Human 3'-phosphoadenosine 5'phosphosulfate (PAPS) synthase: biochemistry, molecular biology and genetic deficiency. IUBMB Life 55: 1-11.

4. Negishi M, Pedersen LG, Petrotchenko E, Shevtsov S, Gorokhov A et al. (2001) Structure and function of sulfotransferases. Arch Biochem Biophys 390: 149-57.

5. Stipanuk MH (2004) Sulfur amino acid metabolism: Pathways for the production and removal of homocysteine and cysteine. Ann Rev Nut 24: 539-577.

6. Minnich V, Smith MB, Brauner MJ, Majerus PW (1971) Glutathione biosynthesis in human erythrocytes I. Identification of the enzymes of glutathione synthesis in hemolysates. J Clin Invest 50: 507-513.

7. Martin JL (1995) Thioredoxin - a fold for all reasons. Structure 3: 245-250.

8. Chesney RW (1985) Taurine: its biological role and clinical implications Adv in Pediat 32: 1-42.

9. Lertratanangkoon K, Wu CJ, Savaraj N, Thomas ML (1997) Alteration of DNA methylation by glutathione depletion. Cancer Letters 120: 149-156.

10. Finkelstein JD (1990) Methionine metabolism in mammals. J of Nut Biochem (1) 228-237.

11. Cantoni GL (1953) S-Adenosylmethionine: A new intermediate formed enzymatically from L-methionine and adenosinetriphosphate. J Biol Chem 204: 403-416.

12. Cantoni GL and Durell J (1957) Activation of methionine for transmethylation. II. The methionine-activating enzyme: studies on the mechanism of reaction. J Biol Chem 225: 1033-1048.

13. Chiang PK, Gordon RK, Zeng GC, Doctor BP, Pardhasarathi K, McCann PP (1996) S-adenosyl methionine and methylation. FASEB J 10: 471-480.

14. Nakagawa $\mathrm{H}$ and Kimura $\mathrm{H}$ (1968) Purification and properties of cystathionine synthetase from rat liver: separation of cystathionine synthetase from serine dehydratase. Biochem Biophys Res Commun 32: 209-214.

15. Clarke S, Banfield K (2001) S-adenosylmethionine dependent methyltransferases (Homocyeteine in health and diseases Edited: Camel R, Acobser DW, Cambridge University Press) Chapter 7: 63-78.

16. Flavin $M$ and Segal A (1964) Purification and properties of the cystathionine $\gamma$-cleavage enzyme of Neurospora. J Biol Chem 239: 2220-2227.

17. Foster MA, Dilworth MJ, Woods DD (1964) Cobalamin and the synthesis of methionine by Escherichia coli. Nature 201: 39-42.

18. Guest JR, Friedman S, Foster MA, Tejerina G, Woods DD (1964) Transfer of the methyl group from N5-methyltetrahydrofolates to homocysteine in Escherichia coli. Biochem J 92: 497-504.

19. Holliday R (1990) DNA Methylation and Epigenetic Inheritance. Philosophical Transactions of the Royal Society of London. Series B, Biological Sciences 326: 329-338.

20. Katoh Y, Ikura T, Hoshikawa Y, Tashiro S, Ito T, Ohta M, Kera Y, Noda $\mathrm{T}$, Igarashi $\mathrm{K}$ (2011) Methionineadenosyltransferase II serves as a transcriptional corepressor of Maf oncoprotein. Molecular Cell 41: 554-566.

21. Faria AF, Laubach H, Venkatachalam KV (2013) Methionine $\gamma$-Lyase 2AminoButyrate Deaminase (MEGL-2ABD) as a Gene Therapeutic Agent for Cancers. J Genet Syndr Gene Ther 4:125-130.

22. Selhub J, Miller JW (1992) The pathogenesis of homocysteinemia: Interruption of the coordinate regulation by S-adenosylmethionine of remethylation and transsulfurylation of homocysteine. Am. Soc. Clin. Nutr. 55: 138-138.

23. Fraga MF, Ballestar E, Paz MF et. al. (2005) Epigenetic differences arise during the lifetime of monozygotic twins. PNAS 102: 10604-10609.

24. Kaminsky ZA, Tang T, Wang SC et. al. (2009) DNA methylation profiles in monozygotic and dizygotic twins. Nat Genet 41: 240-245.

25. Fell R, Fraga MF (2012) Epigenetics and the environment: emerging patterns and implications. Genetics 13: 97-109. 
Citation: Venkatachalam KV (2014) Methionine Metabolism in Humans: New Perspectives on Epigenetic Inheritance. J Mol Genet Med 8: 138. doi:10.4172/1747-0862.1000138

Page 4 of 4

26. Kim YI (2005) Nutritional Epigenetics: Impact of folate deficiency on DNA methylation and colon cancer susceptibility. J. Nutr. 135: 2703-2709.

27. Jayanta C, Frederick W. (2004) Class-switch recombination: interplay of transcription, DNA deamination and DNA repair. Nat Rev Immun 4: 541-552.
28. Fortini P, Dogliotti E (2007) Base damage and single-strand break repair: mechanisms and functional significance of short- and long-patch repair subpathways. DNA Repair 6: 398-409. 